\title{
Identification of SARS-CoV2-mediated suppression of NRF2 signaling reveals a potent antiviral and anti- inflammatory activity of 4-octyl-itaconate and dimethyl fumarate
}

\section{David P. Olagnier ( $\square$ olagnier@biomed.au.dk)}

Department of Biomedicine, Aarhus Research Center for Innate Immunology, Aarhus University, Aarhus, Denmark

\section{Ensieh Farahani}

Department of Biomedicine, Aarhus Research Center for Innate Immunology, Aarhus University, Aarhus, Denmark

\section{Jacob Thyrsted}

Department of Biomedicine, Aarhus Research Center for Innate Immunology, Aarhus University, Aarhus, Denmark

\section{Julia B. Cadanet}

Department of Biomedicine, Aarhus Research Center for Innate Immunology, Aarhus University, Aarhus, Denmark

\section{Angela Herengt}

Department of Biomedicine, Aarhus Research Center for Innate Immunology, Aarhus University, Aarhus, Denmark

\section{Manja Idorn}

Department of Biomedicine, Aarhus Research Center for Innate Immunology, Aarhus University, Aarhus, Denmark

\section{Alon Hait}

Department of Infectious Diseases, Aarhus University Hospital, Aarhus, Denmark

\section{Bruno Hernaez}

Centro de Biología Molecular Severo Ochoa (Consejo Superior de Investigaciones Científicas Universidad Autónoma de Madrid), Nicolás Cabrera 1, 28049 Madrid, Spain.

\section{Alice Knudsen}

Department of Biomedicine, Aarhus Research Center for Innate Immunology, Aarhus University, Aarhus, Denmark

\section{Marie Beck Iversen}

1Department of Biomedicine, Aarhus Research Center for Innate Immunology, Aarhus University, Aarhus, Denmark

\section{Mirjam Schilling}


3Medical Research Council Human Immunology Unit, Medical Research Council Weatherall Institute of Molecular Medicine, Radcliffe Department of Medicine, University of Oxford, Oxford OX3 9DS, UK

\section{Sofie E. Jørgensen}

Department of Infectious Diseases, Aarhus University Hospital, Aarhus, Denmark

\section{Michelle Thomsen}

Department of Infectious Diseases, Aarhus University Hospital, Aarhus, Denmark

\section{Line Reinert}

Department of Biomedicine, Aarhus Research Center for Innate Immunology, Aarhus University, Aarhus, Denmark

\section{Michael Lappe}

Omiics ApS, Åbogade 15, 8200 Aarhus N, Denmark

\section{Huy-Dung Hoang}

Children's Hospital of Eastern Ontario Research Institute, Department of Biochemistry Microbiology and Immunology, University of Ottawa, Ottawa, Ontario, Canada, K1H 8L1

\section{Victoria H. Gilchrist}

Children's Hospital of Eastern Ontario Research Institute, Department of Biochemistry Microbiology and Immunology, University of Ottawa, Ottawa, Ontario, Canada, K1H 8L1.

\section{Anne Louise Hansen}

Department of Biomedicine, Aarhus Research Center for Innate Immunology, Aarhus University, Aarhus, Denmark

\section{Rasmus Ottosen}

Department of Chemistry, Aarhus University, Aarhus, Denmark

\section{Camilla Gunderstofte}

Department of Biomedicine, Aarhus Research Center for Innate Immunology, Aarhus University, Aarhus, Denmark

\section{Charlotte Møller}

Department of Biomedicine, Aarhus Research Center for Innate Immunology, Aarhus University, Aarhus, Denmark

\section{Jinrong Huang}

Lars Bolund Institute of Regenerative Medicine, BGI-Shenzhen, Shenzhen 518083, China

\section{Martin Jakobsen}

Department of Biomedicine, Aarhus Research Center for Innate Immunology, Aarhus University, Aarhus, Denmark

\section{Thomas B. Poulsen}

Department of Chemistry, Aarhus University, Aarhus, Denmark

\section{Lydia Bartsch}

Department of Pediatrics and Adolescent Medicine, Division of Pediatric Neurology, University Medical Center Göttingen, 37075 Göttingen, Germany.

\section{Anne L. Thielke}


Department of Biomedicine, Aarhus Research Center for Innate Immunology, Aarhus University, Aarhus, Denmark

\section{Yonglun Luo}

Lars Bolund Institute of Regenerative Medicine, BGI-Shenzhen, Shenzhen 518083, China

\section{Tommy Alain}

Children's Hospital of Eastern Ontario Research Institute, Department of Biochemistry Microbiology and Immunology, University of Ottawa, Ottawa, Ontario, Canada, K1H 8L1

\section{Jan Rehwinkel}

Medical Research Council Human Immunology Unit, Medical Research Council Weatherall Institute of Molecular Medicine, Radcliffe Department of Medicine, University of Oxford, Oxford OX3 9DS, UK

\section{Antonio Alcamí}

Centro de Biología Molecular Severo Ochoa (Consejo Superior de Investigaciones Científicas Universidad Autónoma de Madrid), Nicolás Cabrera 1, 28049 Madrid, Spain

\section{John Hiscott}

Istituto Pasteur Italia-Cenci Bolognetti Foundation, Viale Regina Elena 291, 00161, Rome, Italy

\section{Trine Mogensen}

Department of Infectious Diseases, Aarhus University Hospital, Aarhus, Denmark

\section{Søren R. Paludan}

Department of Biomedicine, Aarhus Research Center for Innate Immunology, Aarhus University, Aarhus, Denmark

\section{Christian K. Holm ( $\sim$ holm@biomed.au.dk)}

Department of Biomedicine, Aarhus Research Center for Innate Immunology, Aarhus University, Aarhus, Denmark

\section{Research Article}

Keywords: SARS-CoV2, COVID-19, NRF2 anti-oxidant gene expression pathway, 4-octyl-itaconate, dimethyl fumarate

Posted Date: May 27th, 2020

DOI: https://doi.org/10.21203/rs.3.rs-31855/v1

License: (c) (i) This work is licensed under a Creative Commons Attribution 4.0 International License. Read Full License

Version of Record: A version of this preprint was published at Nature Communications on October 2nd, 2020. See the published version at https://doi.org/10.1038/s41467-020-18764-3. 


\section{Abstract}

Antiviral strategies to inhibit Severe Acute Respiratory Syndrome Coronavirus 2 (SARS-CoV2) and the pathogenic consequences of COVID-19 are urgently required. Here we demonstrate that the NRF2 antioxidant gene expression pathway is suppressed in biopsies obtained from COVID-19 patients. Further, we uncover that NRF2 agonists 4-octyl-itaconate (4-OI) and the clinically approved dimethyl fumarate (DMF) induce a potent cellular anti-viral program, which potently inhibits replication of SARS-CoV2 across cell lines. The anti-viral program extended to inhibit the replication of several other pathogenic viruses including Herpes Simplex Virus-1 and-2, Vaccinia virus, and Zika virus through a type I interferon (IFN)independent mechanism. In addition, induction of NRF2 by 4-OI and DMF limited host inflammatory responses to SARS-CoV2 infection associated with airway COVID-19 pathology. In conclusion, NRF2 agonists 4-OI and DMF induce a distinct IFN-independent antiviral program that is broadly effective in limiting virus replication and suppressing the pro-inflammatory responses of human pathogenic viruses, including SARS-CoV2.

One Sentence Summary: NRF2 agonists 4-octyl-itaconate (4-OI) and dimethyl fumarate inhibited SARSCoV2 replication and virus-induced inflammatory responses, as well as replication of other human pathogenic viruses.

\section{Introduction}

The 2020 SARS-CoV2 pandemic emphasizes the urgent need to identify cellular factors and pathways that can be targeted by new broad-spectrum anti-viral therapies. Viral infections usually cause disease in humans through both direct cytopathogenic effects and through excessive inflammatory responses of the infected host. This also seems to be the case with SARS-CoV2 as COVID-19 patients develop cytokine storms that are very likely to contribute to, if not drive, immunopathology and severe disease $(1,2)$. For these reasons, anti-viral therapies must aim to not only inhibit viral replication but also to limit inflammatory responses of the host.

Nuclear factor (erythroid-derived 2) -like 2 (NRF2) functions as a cap'n'collar basic leucine zipper family of transcription factors characterized structurally by the presence of NRF2-ECH homology domains(3). At homeostasis, NRF2 is kept inactive in the cytosol by its inhibitor protein KEAP1 (Kelch-like ECHassociated protein 1), which targets NRF2 for proteasomal degradation(4). In response to oxidative stress, KEAP1 is inactivated and NRF2 is released to induce NRF2-responsive genes. In general, the genes under the control of NRF2 protect against stress-induced cell death and NRF2 has thus been suggested as the master regulator of tissue damage during infection(5). Importantly, NRF2 is now demonstrated as an important regulator of the inflammatory response $(6,7)$ and functions as a transcriptional repressor of inflammatory genes, most notably interleukin (IL-) 1b, in murine macrophages(8). Recent reports have now demonstrated that NRF2 is induced by several cell derived metabolites including itaconate and 
fumarate, to limit inflammatory responses to stimulation of TLR signaling with lipopolysaccharide stimulation(9). The chemically synthesized and cell-permeable derivative of itaconate, 4-octyl-itaconate (4-OI) was then demonstrated to be a very potent NRF2 inducer(9). Of special interest is the derivative of fumarate, dimethyl fumarate (DMF), a US Food and Drug Administration (FDA) approved drug, which is used as an anti-inflammatory therapeutic in multiple sclerosis (MS) and demonstrated, at least in animal models, a potent capacity to suppress pathogenic inflammation through a Nrf2-dependent mechanism $(10,17)$.

Besides limiting the inflammatory response to LPS, induction of NRF2 by 4-OI also inhibits the Stimulator of Interferon Genes (STING) antiviral pathway along with interferon (IFN) stimulated gene expression(12). In opposition to this anti-viral effect of NRF2 on the IFN-response a recent single-cell RNA-seq analysis has demonstrated that NRF2 gene expression signatures correlated negatively with susceptibility to HSV1 infection (13). If NRF2 agonists can be used to limit viral replication of SARS-CoV2 or other pathogenic viruses is, however, not known.

Here we demonstrate that expression of NRF2-dependent genes is suppressed in biopsies from COVID-19 patients and that treatment of cells with NRF2 agonists 4-OI and DMF induces a strong anti-viral program that limits SARS-CoV2 replication. The anti-viral effect of activating NRF2 extended to other pathogenic viruses including Herpes Simplex Virus-1 and-2 (HSV-1 and HSV-2), Vaccinia Virus (VACV), and Zika Virus (ZIKV). Further, 4-OI and DMF limited the release of pro-inflammatory cytokines in response to SARSCoV2 infection and to virus-derived ligands through a mechanism that limits IRF3 dimerization. In summary, we demonstrate that NRF2 agonists are plausible broad-spectrum anti-viral and antiinflammatory agents and we suggest a repurposing of the already clinically approved DMF for the treatment of SARS-CoV2.

\section{Results}

\section{NRF2 dependent anti-oxidant response is suppressed in COVID-19 patient biopsies}

To identify host factors or pathways that are important for controlling SARS-CoV2 infection, publicly available transcriptome data sets including transcriptome analysis of lung biopsies from COVID-19 patients were analyzed using differential expression analysis. As shown in Fig. 1a-d, genes linked with inflammatory and anti-viral pathways, including RIG-I receptor and Toll-like receptor signaling, were highly enriched in COVID-19 patient samples, whereas genes associated with the NRF2 dependent anti-oxidant response were highly suppressed. These data indicate that SARS-CoV2 targets the anti-oxidant NRF2 pathway and thus suggests that the NRF2 pathway restricts SARS-CoV2 replication.

NRF2 agonists 4-OI and dimethyl fumarate are strong inhibitors of SARS-CoV2 replication 
Considering that NRF2 suppresses anti-viral IFN-responses, it was surprising to discover that treatment of Vero cells with 4-OI generated a peak 5-log reduction in SARS-CoV2 RNA levels in a dose dependent manner (Fig. 2a+b). Further, subsequent release of progeny SARS-CoV2 virus particles to the cell supernatant was equally decreased by 4-OI treatment as measured by TCID50 assay and plaque assay

(Fig. 2c-f). The reduced viral replication led to reduced virus-induced cytotoxicity of the infected Vero cells determined by lactate dehydrogenase release assay and by immunoblotting for cleaved Caspase 3 and Poly(ADP-Ribose) Polymerase 1 (PARP-1), which are hallmark indicators of apoptosis(14) (Fig, $\mathbf{2} \mathbf{g}+\mathbf{h}$ ). Interestingly, the observation that Nrf2 pathway is inhibited in response to SARS-CoV-2 infection could be recapitulated in SARS-CoV-2 infected Vero cells as demonstrated by both the basal decrease in Nrf2driven proteins $\mathrm{HO}-1$ and $\mathrm{NqO} 1$ and their incapacity to be induced by Nrf2 agonist 4-OI (Fig. 2h). The effect of 4-OI was also retained in the lung cancer cell line Calu-3, where SARS-CoV2 RNA levels were reduced by $>2$-logs (Fig. 2i), while release of progeny virus was reduced by $>6$-logs based on TCID50 analysis of cell supernatants (Fig. $\mathbf{2 j + k}$ ). In the immortalized human epithelial cell line NuLi total infection levels were relatively low compared to what we could observe in Calu3 and Vero cells but 4-OI treatment still reduced SARS-CoV2 RNA levels by approximately 50-fold (Fig. 2l+m). We further tested the anti-viral effect towards SARS-CoV2 in primary human airway epithelial (HAE) cultures (Fig. 2n). Here, 4-OI treatment also significantly reduced viral RNA levels (Fig. 20). Interestingly, when treating Calu3 cells with DMF, a known NRF2 inducer and clinically approved drug in the first-line-of treatment of multiple sclerosis, we could observe an anti-viral effect toward SARS-CoV2 replication similar in magnitude as what we had observed with 4-OI (Fig 2p) as well as a significant effect when using Vero cells (Fig. 2q). These data demonstrate that NRF2 inducers 4-OI and DMF induce potent anti-viral responses that efficiently inhibit SARS-CoV2 replication across multiple cellular systems.

\section{Activation of NRF2 with 4-OI broadly inhibits viral replication through an IFN-independent pathway}

The anti-viral effect of 4-OI was not restricted to SARS-CoV2 but also extended to other human pathogenic viruses. Using the human keratinocyte cell line HaCaT as a model of Herpes Simplex Virus (HSV) type 1 and 2 we could observe that treatment with 4-Ol reduced both the release of progeny virus, the cellular content of virus RNA determined by RNA sequence analysis, and viral protein as determined by both immunoblotting and flow cytometry (Fig. 3a-e and Fig. S1). By contrast, the expression of NRF2inducible genes Heme Oxygenase 1 (HO-1), NAD(P)H dehydrogenase 1 (Nq01), and Sequestosome 1 (SQSTM1) was highly increased in response to 4-OI treatment (Fig. $\mathbf{2}$ and Fig. S1). The anti-viral effect of 4-OI was dependent on NRF2 as silencing hereof by siRNA clearly reduced the suppression of HSV1 infection by 4-OI (Fig. 2f-g). 
Vaccinia virus (VACV) belongs to the family of human pathogenic poxviruses. We used HaCaT cells, but also bone marrow derived dendritic cells (BMDCs), to test if the anti-viral effect of 4-Ol extended to these viruses. Here we could observe that both $\mathrm{HaCaT}$ cells and BMDCs became highly resistant to infection with VACV when these were pre-treated with 4-OI as measured by plaque assay and flow cytometry (Fig. 2h-I). For both HSV1 and VACV the anti-viral effect of 4-Ol was extended to other cell type including murine cancer cell line 4T1 and human renal carcinoma 786-0 cells (Fig. S2). Interestingly, the anti-viral effect of 4-Ol was not extended to infection with vesicular stomatitis virus (VSV) emphasizing that the anti-viral program induced by 4-Ol effectively inhibits replication of many, but not all, viruses (Fig. S2). The anti-viral effect of 4-OI relied on intracellular restriction of replication, since viral entry was not affected by 4-OI treatment - if anything it seemed to be slightly increased (Fig. S3).

To determine if the anti-viral effect of 4-Ol extended to an in vivo model of viral pathogenesis, female C57BL6J mice were treated with 4-OI prior to vaginal inoculation with HSV; pre-treatment with 4-OI decreased disease progression (Fig. S4), an effect that was enhanced in mice deficient in STING $\left(\right.$ TMEM173 ${ }^{-/-}$) most likely to due to the pro-viral effect 4-OI has on the STING signaling pathway $(12,15)$, which is eliminated in these mice.

Finally, we tested the efficacy of 4-OI on Zika virus, an important human pathogenic virus causing mild symptoms in the competent adult but severe disease when transmitted in utero(16). Here, we could demonstrate that the anti-viral program induced by 4-OI reduced replication of Zika virus in the human lung cancer cell line A549 and in the human liver cell line Huh-7 (Fig. 2m-n). Given that Vero cells are deficient in type I IFN(17), this suggested that the inhibitory effect of 4-OI was actually independent of type I IFN signaling. To address this possibility, we used either HaCaT cells, deficient in Signal Transducer and Activator of Transcription 1 (STAT1), which is necessary for type I IFN-signaling(18); or deficient in STING, which is central to type I IFN-response to DNA viruses. Here, cells were treated with 4-OI, followed by infection with HSV1 and VACV. Replication of both viruses was inhibited by 4-OI in STAT1 KO cells and for HSV1 also in STING KO cells as measured by plaque assay and expression of viral proteins by immunoblotting and flow cytometry (Fig. S5). In conclusion, 4-OI induces an NRF2 mediated anti-viral program that operates independently of IFN signaling (Fig. S5).

\section{4-OI and DMF suppress the inflammatory response to SARS-CoV2}

In COVID-19, an uncontrolled pro-inflammatory cytokine storm contributes to disease pathogenesis and lung damage (19). For this reason, we investigated if 4-OI and DMF could inhibit expression of pro- 
inflammatory cytokines induced by SARS-CoV2. In Calu-3 cells, infection with SARS-CoV2 increased the expression of IFNB1, C-X-C motif chemokine 10 (CXCL10), Tumor Necrosis Factor alpha (TNFA), IL-1B and $\mathrm{C}-\mathrm{C}$ chemokine ligand 5 (CCL5). Interestingly, this was abolished by pretreatment with 4-OI thus severely reducing the pro-inflammatory response to SARS-CoV2 (Fig. 4a-b). By contrast, expression of the NRF2 inducible gene HMOX1 was highly increased in response to 4-Ol treatment (Fig. 4c). The potential anti-inflammatory effect of 4-OI in this context was supported when using HAE cultures. Here, treatment with 4-OI also reduced the expression of IFNB1, CXCL 10, TNFa, and CCL5 in the context of SARS-CoV2 infection (Fig. 4d-e), while increasing the expression of the NRF2 inducible gene HMOX1 (Fig. 4f). A similar pattern was seen in experiments where Calu3 cells were treated with DMF before SARS-CoV2 infection. Here, IFNB1, CXCL 10 and CCL 5 mRNA levels were highly reduced in DMF treated cells while TNFA mRNA levels seemed unaffected (Fig. $\mathbf{4 g}+\mathbf{h}$ ). By contrast, treatment with DMF increased the mRNA expression levels of NRF2 inducible gene HMOX1 (Fig. 4i). As inflammatory responses often stem from immune cells we also tested the effect of 4-OI on Peripheral Blood Mononuclear Cells (PBMCs) harvested from healthy donors. Although stimulation of PBMCs with SARS-CoV2 yielded a very weak induction of CXCL10 compared to sendai virus (SeV) infection, and no detectable induction of other cytokines, 4-OI treatment also reduced CXCL10 mRNA levels in this context (Fig. 4j). Further, when using PBMCs harvested from four individual patients with severe COVID-19 and admitted to hospital Intensive Care Units (ICUs), we could conclude that in three out of four patients, expression levels of CXCL10 were increased when compared to healthy controls; and that in all four patients, these levels were strongly reduced to or below normal when treating the PBMCs with 4-OI (Fig. 4k) indicating that 4-Ol is able to relieve inflammatory responses induced by SARS-CoV2 in PBMCs in vivo.

The observed decrease in anti-viral and pro-inflammatory responses could possibly be explained by the 4OI mediated reduction in cellular viral RNA with subsequent reduced induction of cytokines through cellular RNA sensors such as RIG-I. We therefore investigated the effect of 4-OI on the induction of IFN and of IFN stimulated genes (ISGs) responses activated by a sequence optimized RIG-I agonist M8(20). Interestingly, 4-OI treatment reduced IFN-responses induced by this RIG-I agonist M8 (Fig 4I-m), through an effect linked to the inhibition of Interferon Regulatory Factor 3 (IRF3) dimerization but not of upstream phosphorylation of Tank Binding Kinase 1 (TBK1) or of IRF3 expression itself (Fig 4n). Importantly, NRF2 expression itself was closely associated with the inhibition of IRF3 dimerization and host antiviral gene expression, since NRF2 silencing by siRNA was sufficient to restore IRF3 dimerization and limit the inhibitory effect of 4-OI (Fig. $4 \mathbf{n}-0$ ). When using the constitutively active form of IRF3, IRF3(5D) (21), 4-OI was still able to block IRF3 dimerization, and again, this effect was eliminated when NRF2 expression was suppressed by siRNA (Fig 4p-q). These data indicate that an NRF2 inducible and dependent mechanism targets the induction of IFN by inhibition of IRF3 dimerization. This phenomenon is likely to add to the inhibition of SARS-CoV2 induced cytokine release we could observe when using NRF2 agonists. We have previously reported that 4-OI inhibits the expression of STING, which is important for the induction of the IFN-response in cells stimulated with cytosolic DNA(12). In line, 4-OI inhibited the IFNresponse to HSV1 infection and to stimulation with STING agonists dsDNA and cGAMP (Fig. 4R-T). 


\section{Discussion}

Altogether, this study demonstrated that the expression of NRF2 dependent anti-oxidant genes was significantly inhibited in COVID-19 patients, and that the NRF2 agonists 4-OI and DMF inhibited both SARS-CoV2 replication, as well as the expression of associated inflammatory markers. The ability of these NRF2 inducers to also reduce potentially pathogenic IFN- and inflammatory responses while retaining their anti-viral properties is unique to these compounds and promotes their applicability to prevent virus-induced pathology. As DMF is currently used as an anti-inflammatory drug in relapsingremitting MS, this drug could be easily repurposed and tested in clinical trials to test of its ability to limit SARS-CoV2 replication and inflammation-induced pathology in COVID-19 patients. Our observation that 4-Ol strongly inhibits the IFN-response to both cytosolic DNA and d RNA, which are canonical anti-viral pathways, but still retain its ability to block viral replication also suggests a spectrum of unidentified cellular programs that are inducible through NRF2 and efficiently restrict viral replication independently of IFNs. This is supported by already mentioned negative correlation between expression of NRF2-inducible genes and infection with HSV1 discovered by Wyler et al., through single cell transcriptome analysis. Similarly to how IFNs block viral replication through the induction of hundreds of effector IFN-stimulated effector genes, the NRF2 controlled anti-viral program might also consists of a myriad of mechanisms that restrict viral replication each by targeting distinct stages of viral replication.

Future studies will determine if patients developing severe SARS-CoV2 pathology also have an underlying NRF2 deficiency, leading to reduced control of viral replication, coupled with excess inflammatory responses. Further, it could be valuable to investigate if patients already in DMF therapy have altered susceptibility to SARS-CoV2 infection and if those infected have milder symptoms and reduced cytokine load. Finally, the fact that 4-Ol effectively limited replication of several human pathogenic viruses demonstrated that 4-OI, or related chemically modified compounds such as DMF, could be evaluated as broad-spectrum anti-viral agents for protection against seasonal and pandemic viral infections in general.

\section{Declarations}

Funding: This research work was supported by Ester M og Konrad Kristian Sigurdssons Dyreværnsfond, Beckett-Fonden, Kong Christian IX og Dronning Louises Jubilæumslegat, Læge Sofus Carl Emil Friis og Hustru Olga Doris Friis' legat, Købmand I Odense Johan og Hanne Weimann Født Seedorffs Legat, UK Medical Research Council (MRC core funding of the MRC Human Immunology Unit; JR), Lundbeck foundation (R303-2018-3379 and R219-2016-878, and R268-2016-3927), and Independent Research Fund Denmark - Medical Sciences (9039-00078B, 4004-00047B, and 0214-00001B). CarlsbergFoundation (Semper Ardens) and European Research Council (ERC-AdG ENVISION; 786602). Marie Skłodowska-Curie Action of the European Commission \# 813343 and Italian Cancer Research Society \#22891 to JH.

Author contributions: $\mathrm{DO}$ and $\mathrm{CH}$ conceived the project and prepared figures. DO, ALH, JT, JC, BH, MI, AK, LR, MI, MS, SF, MT, ML, HH, VG, AH, AK, CG, CM, LB, AT, TA, JH, RO, and AA performed experiments, 
analyzed data, and prepared figures. EF performed bioinformatics and prepared figures. TM was responsible for including COVID-19 patients and achieving patient material. TA, JR, AA, TM, SP, and CH planned experiments and analyzed data. $\mathrm{CH}$ drafted and finalized the manuscript. JH planned experiments and edited the manuscript. EF and ML performed bioinformatics and prepared figures. MJ facilitated SARS-CoV2 laboratories. Authors declare no competing interests. Data and materials availability: All data is available in the main text or the supplementary materials,

\section{References}

1. P. Mehta et al., COVID-19: consider cytokine storm syndromes and immunosuppression. Lancet 395, 1033-1034 (2020).

2. C. Huang et al., Clinical features of patients infected with 2019 novel coronavirus in Wuhan, China. Lancet 395, 497-506 (2020).

3. G. P. Sykiotis, D. Bohmann, Stress-activated cap'n'collar transcription factors in aging and human disease. Science signaling 3, re3 (2010).

4. J. D. Hayes, A. T. Dinkova-Kostova, The Nrf2 regulatory network provides an interface between redox and intermediary metabolism. Trends Biochem Sci 39, 199-218 (2014).

5. M. P. Soares, A. M. Ribeiro, Nrf2 as a master regulator of tissue damage control and disease tolerance to infection. Biochem Soc Trans 43, 663-668 (2015).

6. R. K. Thimmulappa et al., Nrf2 is a critical regulator of the innate immune response and survival during experimental sepsis. J. Clin. Invest. 116, 984-995 (2006).

7. R. K. Thimmulappa et al., Nrf2-dependent protection from LPS induced inflammatory response and mortality by CDDO-Imidazolide. Biochem. Biophys. Res. Commun. 351, 883-889 (2006).

8. E. H. Kobayashi et al., Nrf2 suppresses macrophage inflammatory response by blocking proinflammatory cytokine transcription. Nature communications 7, 11624 (2016).

9. E. L. Mills et al., Itaconate is an anti-inflammatory metabolite that activates Nrf2 via alkylation of KEAP1. Nature 556, 113-117 (2018).

10. R. Gold, R. A. Linker, M. Stangel, Fumaric acid and its esters: an emerging treatment for multiple sclerosis with antioxidative mechanism of action. Clin. Immunol. 142, 44-48 (2012).

11. R. A. Linker et al., Fumaric acid esters exert neuroprotective effects in neuroinflammation via activation of the Nrf2 antioxidant pathway. Brain 134, 678-692 (2011).

12. D. Olagnier et al., Nrf2 negatively regulates STING indicating a link between antiviral sensing and metabolic reprogramming. Nature communications 9, 3506 (2018).

13. E. Wyler et al., Single-cell RNA-sequencing of herpes simplex virus 1-infected cells connects NRF2 activation to an antiviral program. Nature communications 10, 4878 (2019). 
14. G. V. Chaitanya, A. J. Steven, P. P. Babu, PARP-1 cleavage fragments: signatures of cell-death proteases in neurodegeneration. Cell Commun Signal 8, 31 (2010).

15. C. Gunderstofte et al., Nrf2 Negatively Regulates Type I Interferon Responses and Increases Susceptibility to Herpes Genital Infection in Mice. Front Immunol 10, 2101 (2019).

16. A. Fajardo, J. Cristina, P. Moreno, Emergence and Spreading Potential of Zika Virus. Front Microbio/7, 1667 (2016).

17. J. Desmyter, J. L. Melnick, W. E. Rawls, Defectiveness of interferon production and of rubella virus interference in a line of African green monkey kidney cells (Vero). J. Virol. 2, 955-961 (1968).

18. L. B. Ivashkiv, L. T. Donlin, Regulation of type I interferon responses. Nat Rev Immuno/ 14, 36-49 (2014).

19. S. Davidson, S. Crotta, T. M. McCabe, A. Wack, Pathogenic potential of interferon alphabeta in acute influenza infection. Nature communications 5, 3864 (2014).

20. L. Castiello et al., An optimized retinoic acid-inducible gene I agonist M8 induces immunogenic cell death markers in human cancer cells and dendritic cell activation. Cancer Immunol. Immunother. 68, 1479-1492 (2019).

21. R. Lin, Y. Mamane, J. Hiscott, Structural and functional analysis of interferon regulatory factor 3 : localization of the transactivation and autoinhibitory domains. Mol. Cell. Biol. 19, 2465-2474 (1999).

\section{Material And Methods}

Cell lines, reagents and culture conditions

Human lung adenocarcinoma epithelial A549 cells, immortalized human HaCaT keratinocytes, Calu-3 epithelial lung cancer cells and human embryonic kidney HEK293T cells were kindly provided by Søren R. Paludan (Aarhus University, Denmark) and cultured in DMEM (Lonza) supplemented with $10 \%$ heat inactivated fetal calf serum, $200 \mathrm{IU} \cdot \mathrm{mL}^{-1}$ penicillin, $100 \mathrm{mg} \cdot \mathrm{mL}^{-1}$ streptomycin and $600 \mathrm{mg} \cdot \mathrm{mL}^{-1}$ glutamine (hereafter termed DMEM complete). Vero E6 cells expressing hTMPRSS2 were a kind gift of Makoto Takeda (University of Tokyo, Japan)(1) and were cultured in DMEM (Lonza) supplemented with $10 \%$ heat inactivated fetal calf serum, $200 \mathrm{IU} \cdot \mathrm{mL}^{-1}$ penicillin, $100 \mathrm{mg} \cdot \mathrm{mL}^{-1}$ streptomycin, $600 \mathrm{mg} \cdot \mathrm{mL}^{-1}$ glutamine and $10 \mathrm{mg}^{\mathrm{mL}} \mathrm{L}^{-1}$ blasticidin. All cell lines were regularly tested for mycoplasma contamination by sequencing from GATC Biotech (Germany). 4-octyl-itaconate (4-OI) was chemically synthetized by Thomas B. Poulsen (Aarhus University, Denmark) and was dissolved in DMSO as previously described here(2).

To obtain bone marrow-derived cells (BMDCs), a cell suspension from femurs of C57BL/ 6 mice (Charles River) was cultured for $6-8$ days at $37^{\circ} \mathrm{C}$ in RPMI- 1640 medium supplemented with $3 \% \mathrm{FBS}$ and $10 \%$ of J558 cell line supernatant containing GM-CSF. Cells were seeded at a density of $10^{6} \mathrm{cells} / \mathrm{ml}$ and medium 
was partially replaced every 2 days. BSC-1 cells (ECACC) were maintained in DMEM supplemented with $5 \%$ FBS,

$2 \mathrm{mM}$ glutamine, $200 \mathrm{IU} / \mathrm{ml}$ penicillin and $100 \mathrm{IU} / \mathrm{ml}$ streptomycin at $37^{\circ} \mathrm{C} 5 \% \mathrm{CO} 2$.

For generation of KO cell line clones in HaCaT cells specific guide RNA sequences targeting STING (5'AGAGCACACTCTCCGGTACC-3') or STAT1 (5'-TTAATGATGAACTAGTGGAG-3') were cloned into the plasmids pX461 (Addgene) (STING) or LentiCRISPR v2 (Addgene) (STAT1). Wildtype HaCaT cells were transfected with the plasmids using the Lipofectamine 2000 Reagent (Invitrogen, Life Tecnologies). $72 \mathrm{~h}$ post transfection, the GFP expressing cells were sorted as single cells by FACS and clones were grown to larger cultures (STING). Or $24 \mathrm{~h}$ post transfection, the cells were seeded in a dilution sufficient to obtain single cells clones after the puromycin selection. The $2 \mu \mathrm{g} \mathrm{mL}^{-1}$ puromycin selection was initiated $48 \mathrm{~h}$ post transfection and continued for $72 \mathrm{~h}$ (STAT1). Hereafter, single cell clones were grown to larger cultures which were validated for absence of protein by western blotting and functional analysis to confirm the biological effect of the gene deficiency.

\section{$\underline{\text { Viruses }}$}

We used the SARS-CoV2 strain \#291.3 FR-4286 isolated from a patient in Germany, and kindly donated by professor Georg Kochs (Freiburg). The virus was propagated in Vero-TMPRSS2 cells(3). Validation and SARS-CoV2 genome detection was performed with Taqman based qPCR using SARS-CoV2 specific primers and probes with the following sequences: Forward primer: AAATTTTGGGGACCAGGAAC, reverse primer: TGGCACCTGTGTAGGTCAAC, Probe: FAM-ATGTCGCGCATTGGCATGGA-BHQ. HSV-1 KOS strain expressing GFP (HSV-1-GFP), HSV-2 333 strain and HSV-2 MS strain were kindly provided by Søren R. Paludan (Aarhus University, Aarhus, Denmark). All HSVs were propagated in Vero cells, purified by ultracentrifugation, and titrated by standard plaque assay as previously described (4). HaCat cells were infected with the different HSVs at a multiplicity of infection (MOI) of 0.01 in a small volume of serumfree medium for $1 \mathrm{~h}$ at $37^{\circ} \mathrm{C}$. Prior to analysis, cells were incubated with DMEM complete for an additional day of culture. VACV Western reserve strain (VACV-WR) was a recombinant vaccinia virus (VACV) named vtag2GFP expressing the tag2GFP under the control of strong synthetic VACV early/late promoter was kindly provided by Dr. Rafael Blasco (INIA, Spain). VACV-WR and vtag2GFP stocks were semi-purified by centrifugation through a $36 \%$ sucrose cushion and titrated twice by plaque assay. The Brazilian ZIKV isolate ZIKV/H.sapiens/Brazil/PE243/2015 was originally described in (5) and was grown on Vero cells. Viral titers were determined by plaque assay on A549 BVDV NPro cells (kind gift from R. Randall, St Andrews). These cells are optimized for virus growth as they stably express the NPro protein of bovine viral diarrhea virus (BVDV), which induces degradation of IRF3 (6). 
Viral entry assay.

Quantification of HSV-1 entry in the presence of 4-OI was performed using the cold binding assay previously described(7). Cells were pretreated with 4-OI $(150 \mu \mathrm{M})$ or DMSO (control) for 48 hours. Cells were pre-incubated at $4^{\circ} \mathrm{C}$ for 30 minutes, then incubated with HSV-1 at a MOI of 10 for 1 hours at $4^{\circ} \mathrm{C}$. Cells were then shifted to $37^{\circ} \mathrm{C}$ for 1 hours to activate virus internalization. After that, cells were washed twice with PBS, then uninternalized virus particles were washed with citric acid buffer $(135 \mathrm{mM} \mathrm{NaCl}, 10$ $\mathrm{mM} \mathrm{KCl}, 40 \mathrm{mM}$ citric acid, $\mathrm{pH}$ 3) incubation for 5 minutes, then cells were washed twice more with PBS. Cells were scraped and genomic DNA was extracted using QIAamp DNA mini kit (QIAGEN). Quantitative PCR were performed using UL30-F and UL30-R primers for HSV-1 genomic DNA. Primer sequence: UL30F: ACATCATCAACTTCGACTGG, UL30-R: CTCAGGTCCTTCTTCTTGTCC

\section{Primary cells and culture conditions}

Peripheral Blood Mononuclear cells (PBMCs) were isolated from healthy donors (blood donors gave written consent as accordingly to the ethical guidelines at Aarhus University Hospital) by Ficoll Paque gradient centrifugation (GE Healthcare). Monocytes were separated using a monocyte enrichment kit (STEMCELL) according to the manufacturer's instructions or from PBMCs by adherence to plastic in RPMI 1640 supplemented with $10 \%$ AB-positive human serum. Differentiation of monocytes to macrophages was achieved by culturing in Dulbecco's Modified Eagle Medium (DMEM) supplemented with $10 \%$ heat inactivated $A B$-positive human serum 9 days in the presence of $10 \mathrm{ng} / \mathrm{ml} \mathrm{M}-\mathrm{CSF}$ (R\&D Systems), as previously reported in (8).

\section{Patients included in this study.}

All patients were positive for SARS-CoV2 by PCR from throat swab and admitted to the ICU and receiving ventilatory support due to severe pneumonia with a component of acute respiratory distress syndrome (ARDS). P1 27-years old male, day 14 at in ICU. P2 57-year-old female, day 9 in ICU. P3 43 years-old male, day 5 in ICU. P4 28 years-old female, day 3 in ICU.

\section{Air-Liquid Interface Epithelium model}

Primary nasal cells were isolated using a nasal brush (Dent-0-Care, \#620B) inserted into the nasal turbinations and twisted. Cells were isolated from the brush by gently expelling monolayer medium 
(Airway Epithelial Cells Basal Medium, PromoCell, \#C-21260 + 1 pack of Airway Epithelial Cell Growth Medium Supplement, PromoCell, \#39160 + 100 U/ml Penicillin/Streptomycin, Gibco \#10378) and PBS to wash of cells. Cells were cultured in monolayer culture in tissue culture flask (Sarstedt, TC: Standart \#83.3911) coated with $0,1 \mathrm{mg} / \mathrm{ml}$ Bovine type I collagen solution (Sigma-Aldrich, \#804592, diluted in sterile $\mathrm{ddH}_{2} \mathrm{O}$ ). Monolayer cultures were split using $1 \mathrm{x}$ Trypsin mixed with $0.3 \mathrm{mM}$ EDTA (10x Trypsin (2,5 \%), Gibco, \#15090, diluted to working concentration in PBS + UltraPure 0.5 M EDTA, Invitrogen, \#15575) at approx. $80 \%$ confluency. At passage two, cells were seeded at $2-3 \times 10^{\wedge} 4$ cells on $6,5 \mathrm{~mm}$ Transwell membranes (Corning, \#3470) coated with $30 \mathrm{ug} / \mathrm{ml}$ Bovine type I collagen solution (Sigma-Aldrich, \#804592, diluted in sterile $\mathrm{ddH}_{2} \mathrm{O}$ ). Cells were seeded and submerged in 2x P/S (200 U/ml Pen/Strep) DMEM-low glycose (Sigma-Aldrich, D5921) mixed one to one with 2x Monolayer medium (Airway Epithelium Cell Basal Medium, (PromoCell, \#C-21260) supplemented with 2 packs of Airway Epithelial Cell Growth Medium Supplement (PromoCell, \#C-39160) without triiodothyronine $+1 \mathrm{ml}$ of $1.5 \mathrm{mg} / \mathrm{ml}$ BSA). When cultures reach full confluency ALI (=Air-liquid interface) is introduced and medium is changed to ALI medium (Pneumacult ALI medium kit (StemCell, \#5001) with ALI medium supplement (StemCell, \#5001) and $100 \mathrm{U} / \mathrm{ml}$ Pen/strep) supplemented with 24 ug of hydrocortisone (StemCell, \#07925) and $0.2 \mathrm{mg}$ heparin (StemCell, \#07980). Membranes was allowed at least 21 days of differentiation verified by extensive cilia beating and mucus covering.

Upon initiation of treatment ALI cultures was washed for 5 minutes using DMEM (low glycose, no additives) and baso-lateral medium changed for ALI medium containing either 150 uM 4-OI or DMSO. Baso-lateral medium containing treatment was left overnight. 100 ul of DMEM (low glycose) with $150 \mathrm{uM}$ or DMSO was additionally added to the apical compartment overnight. At time of infection, apical medium was removed and 100 ul DMEM (low glycose) containing SARS-CoV-2 at MOI 0.1 was added to all membranes for 1 hour and placed in $37^{\circ} \mathrm{C}$ incubator. After 1-hour apical infection medium was removed and membranes placed in $37^{\circ} \mathrm{C}$ incubator for 24 hours before harvest.

At time of harvest, baso-lateral medium was removed and 500 ul Trypsin/EDTA was added bao-laterally and 200 ul was added apically. After approx. 5 minutes cells were harvested using 5\% FPB/DMEM (low glycose). Cells were lysed for RNA isolation using lysisbuffer from High Pure RNA Isolation Kit (Roche Diagnostics, \#11828665001). For Western blot cells were lysed in RIPA buffer containing 1/10 Protease inhibitor (Roche), 1/1000 Benzonase (Sigma, \#E1014) and 1/50 0.5 M Sodium Flouride.

\section{Short-interfering RNA (siRNA)-mediated knock down}

For short interfering RNA experiments, HaCat cells were transfected in 6-well plates with 80 pmol of human Nrf2(1) (sc-37030) or control si RNA (sc-37007) diluted in serum and antibiotic free DMEM and using Lipofectamine RNAi Max as per manufacturer's instructions. HaCat cells were incubated for $72 \mathrm{~h}$ in the presence of the siRNA before being processed. 
dsDNA, cGAMP and optimized RIG-I agonist stimulation of cells.

HSV-60 naked, a viral dsDNA motif, 2'3'-cGAMP, a STING ligand, and M8, a sequence optimized RIG-I agonist (9) were obtained from Invivogen and John Hiscott (Pasteur Institute, Rome), respectively. Intracellular delivery of dsDNA and cGAMP was achieved using Lipofectamine 2000 (Invitrogen) diluted in serum-free medium with a ratio of Lipo.dsDNA/cGAMP of 1:1. Final concentration for both dsDNA and cGAMP was $4 \mathrm{mg} \mathrm{mL}^{-1}$. Intracellular delivery of M8 was achieved using Lipofectamine RNAiMax (Invitrogen) diluted in serum-free medium with a ratio of Lipo.RNA of 1:1. Final concentration of M8 was $10 \mathrm{ng} \cdot \mathrm{mL}^{-1}$.

\section{$\underline{\text { VACV infection assays. }}$}

BMDCs, HaCaT and HaCaT Stat1 KO were incubated or not with $150 \mu \mathrm{M} 4-\mathrm{Ol}$ for $48 \mathrm{~h}$ before infection with vtag2GFP using 0.1 or $0.01 \mathrm{pfu} / \mathrm{cell}$ at $37^{\circ} \mathrm{C}$ for $60 \mathrm{~min}$. Then, infected cells were washed to remove potential unbound viruses and infection proceeded at $37^{\circ} \mathrm{C}$.

To determine the proportion of vtag2GFP infected cells GFP expression was detected at 16 hpi by flow cytometry using triplicates. Briefly, cells were harvested, washed with FACS buffer (PBS, 0.01\% sodium azide, and $0.1 \% \mathrm{BSA}$ ) and fixed with paraformaldehyde $4 \%$ in PBS for $10 \mathrm{~min}$. After extensive washing with FACS buffer, $2 \times 10^{4}$ cells were scored

and analyzed in a FACSCalibur flow cytometer (BD Sciences) per experimental condition in triplicates. To determine VACV virus titres, HaCaT and HaCaT STAT1 KO cells were previously stimulated for $48 \mathrm{~h}$ or not with $150 \mu \mathrm{M} 4-\mathrm{Ol}$ and then infected with VACV-WR using 0.1 or $0.01 \mathrm{pfu} / \mathrm{cell}$ at $37^{\circ} \mathrm{C}$. At $24 \mathrm{hpi}$, cells were harvested in their own media, centrifuged at $1,800 \times \mathrm{g}$ for $5 \mathrm{~min}$, and resuspended in $0.5 \mathrm{ml}$ of fresh medium. In all cases, samples were frozen, thawed three times and titrated using duplicates in BSC-1 cells. Briefly, preconfluent monolayers of BSC-1 cells were infected with 10-fold serial dilutions of viral inoculums for $1 \mathrm{~h}$ at $37^{\circ} \mathrm{C}$. Then, inoculum was replaced with semi-solid carboxy-methyl cellulose (Sigma) media with $2 \%$ FBS and cells fixed in $10 \%$ formaldehyde at 3 dpi. Plaques were stained with $0.1 \%(\mathrm{w} / \mathrm{v})$ crystal-violet. Two independent experiments were performed.

\section{Zika Virus infections.}

A549 cells (kind gift from G. Kochs, Freiburg) and Huh-7 were cultured at $37^{\circ} \mathrm{C}$ in Dulbecco's Modified Eagle Medium (DMEM), supplemented with 10\% FCS and 2mM L-Glutamine. Cells were seeded in 24 well plates and pre-treated with 4-octyl-itaconate (4-OI) (150uM) for 48h. Cells were infected with ZIKV (moi 0.1 ) for $1 \mathrm{~h}$. 4-Ol was freshly added when the medium was changed.Cells were lysed and total RNA was extracted at 96hpi using the QIAshredder (Qiagen) and RNeasy Mini Kit (Qiagen) according to the manufacturer's instructions. RNA was reverse transcribed using SuperScript II Reverse Transcriptase 
(Invitrogen) into CDNA that was then used for qPCR with SYBR green PCR kit (Life Technologies). $C_{T}$ values were normalized to GAPDH $\left(\Delta C_{T}\right)$. SYBR green primer probes used include GAPDH (for: CATGGCCTTCCGTGTTCCTA, rev: CCTGCTTCACCACCTTCTTGA) and ZIKV (for: CGAGGAACATCCAGACTC, rev: ATTGGAGATCCTGAAGTTCC).

\section{SARS-CoV2 TCDI50\% assay}

The assay was performed as follows. $2 \times 10^{4}$ Vero E6 TMPRSS2 cells were seeded in 90ul DMEM (Gibco, $+2 \%$ FCS (Sigma-aldrich) + 1\% Pen/Strep (Gibco) + L-Glutamine (Sigma-Aldrich) per well in flat-bottom 96-well plates. $24 \mathrm{~h}$ after, samples were titrated onto the cells by addition of $10 \mathrm{ul}$ of a 10 -fold serial dilution. One full plate was used per sample analyzed. Each dilution of supernatant were represented 8 times on a plate. The cells were incubated for $72 \mathrm{~h}$ in a humidified $\mathrm{CO}_{2}$ incubator at $37^{\circ} \mathrm{C}, 5 \% \mathrm{CO}_{2}$, before fixing with $5 \%$ Formalin (Sigma-Aldrich) and staining with crystal violet solution (Sigma-Aldrich). Images were taken using a Leica DMi1, microscope with a Leica MC170 HD camera. TCDI50 \% virus titer calculated by Reed-Muench method.

\section{Western blot.}

HaCat cells were lysed in $100 \mathrm{~mL}$ of ice-cold Pierce RIPA lysis buffer (Thermo Scientific) supplemented with $10 \mathrm{mM} \mathrm{NaF}, 1 \mathrm{x}$ complete protease cocktail inhibitor (Roche) and $5 \mathrm{IU} \cdot \mathrm{mL}^{-1}$ benzonaze (Sigma), respectively. Protein concentration was determined using a BCA protein assay kit (Thermo Scientific). Whole-cell lysates were denatured for $3 \mathrm{~min}$ at $95^{\circ} \mathrm{C}$ in presence of $1 \mathrm{x}$ XT Sample Buffer (BioRad) and $1 \mathrm{x}$ XT reducing agent (BioRad). 10-40 mg of reduced samples was separated by SDS-PAGE on 4-20\% Criterion TGX precast gradient gels (BioRad). Each gel was run initially for $15 \mathrm{~min}$ at $70 \mathrm{~V}$ and $45 \mathrm{~min}$ at 120V. Transfer onto PVDF membranes (BioRad) was done using a Trans-Blot Turbo Transfer system for 7 min. Membranes were blocked for $1 \mathrm{~h}$ with $5 \%$ skim-milk (Sigma Aldrich) at room temperature in PBS supplemented with $0.05 \%$ Tween-20 (PBST). Membranes were fractionated in smaller pieces and probed overnight at $4^{\circ} \mathrm{C}$ with any of the following specific primary antibodies in PBST: anti-Nrf2 (12721, Cell Signaling 1:1000), anti-TBK1/NAK (3013, Cell Signaling 1:1000), anti-phospho-TBK1/NAK (5483, Cell Signaling 1:1000), anti-SQSTM1/p62 (8025, Cell Signaling 1:1000), anti-IRF3 (11904, Cell Signaling 1:1000), anti-phospho-IRF3 (4947, Cell Signaling 1:500), anti-HO-1 (5853, Cell Signaling 1:1000), antiIFIT1 (14769, Cell Signaling 1:1000), anti-NRF2 (12721, Cell Signaling 1:1000), anti-STING (13647, Cell Signaling 1:1000), anti-Nq01 (3187, Cell Signaling 1:1000), and anti-Vinculin (18799, Cell Signaling $1: 1000)$ used as loading control. After three washes in PBST, secondary antibodies, peroxidaseconjugated $F(a b) 2$ donkey anti-mouse Ig $(H+L)$ (1:10000) or peroxidase-conjugated $F(a b) 2$ donkey antirabbit lgG $(\mathrm{H}+\mathrm{L})$ (1:10000) (Jackson ImmunoResearch) were added to the membrane in PBST 1\% milk for $1 \mathrm{~h}$ at room temperature. All membranes were washed three times and exposed using either the 
SuperSignal West Pico PLUS chemiluminescent substrate or the SuperSignal West Femto maximum sensitivity substrate (ThermoScientific) and an Image Quant LAS4000 mini imager (GE Healthcare).

\section{Semi-native WB Dimerization assay.}

IRF3 dimerization was assayed under semi-native conditions. Cells were lysed in ice-cold Pierce RIPA lysis buffer (Thermo Scientific) supplemented with $10 \mathrm{mM} \mathrm{NaF}, 1 \mathrm{x}$ complete protease cocktail inhibitor (Roche) and $5 \mathrm{IU} / \mathrm{mL}$ benzonaze (Sigma). Protein concentration was determined using a BCA protein assay kit (Thermo Scientific). Whole-cell lysates were mixed with 1x XT Sample Buffer (BioRad); samples were neither reduced nor heated before separation was done on 4-20\% Criterion TGX precast gradient gels (BioRad) by SDS-PAGE electrophoresis. Each gel was run initially for $15 \mathrm{~min}$ at $70 \mathrm{~V}$ and $15 \mathrm{~min}$ at $120 \mathrm{~V}$. Transfer onto PVDF membranes (BioRad) was done using a Trans-Blot Turbo Transfer system for 7 min. Membranes were blocked for $1 \mathrm{~h}$ with $5 \%$ skim-milk (Sigma Aldrich) at room temperature in PBS supplemented with $0.05 \%$ Tween-20 (PBST). Membranes were probed overnight at $4^{\circ} \mathrm{C}$ with the following specific primary antibody in PBST: anti-IRF3. After three washes in PBST, secondary antibodies, peroxidase-conjugated $F(a b) 2$ donkey anti-rabbit IgG $(H+L)$ (1:10000) (Jackson Immuno Research) were added to the membrane in PBST $1 \%$ milk for $1 \mathrm{~h}$ at room temperature. All membranes were washed three times and exposed using either the SuperSignal West Pico PLUS chemiluminescent substrate or the SuperSignal West Femto maximum sensitivity substrate (Thermo Scientific).

\section{gPCR analysis.}

Gene expression was determined by real-time quantitative PCR, using TaqMan detection systems (Applied Biosciences). RNA was extracted using the High Pure RNA Isolation kit (Roche) and RNA quality was assessed by Nanodrop spectrometry (Thermo Fisher). RNA levels were analyzed using premade TaqMan assays and the RNA-to-Ct-1-Step kit according to the manufacturer's recommendations (Applied Biosciences).

Transcriptome analysis COVID19

COVID19 data set analysis (Fig. 1): RNA-seq data was obtained from an already available dataset from Blanco-Melo (doi: https://doi.org/10.1101/2020.03.24.004655). From the raw read-counts differential expression values were calculated using DESeq2(10). Significantly differentially expressed genes (SDEGs) were selected based on the thresholds of adjusted P-value $<0.05$ and absolute fold change of 2 (Fig. 1A). In order to focus on commonality across the different conditions with respect to generating 
clinically relevant hypotheses, the 815 SDEGs obtained from the biopsy sample were checked if these were also present in the list of SDEGs in the other conditions. All genes that occur in at least 3 or more other conditions were included in the final list, resulting in 113 genes. Finally, the expression values for all genes in the final list across all conditions were assembled, clustered using Euclidean distance metric and Ward's variance minimization algorithm, and visualized as a heatmap using Python3.7 and seaborn cluster-map tools. Then the gene-sets from each of the outlined clusters were used for pathway enrichment analysis using Enrichr (Fig. 1B) (11). Finally, the STRING database(12) was used to construct the cloud network starting from lists of genes manually annotated for NRF2, inflammation and IFN signaling. Edges and nodes were extracted from STRING and imported to Cytoscape (13) version 3.7 for further visualization (Fig. 1D).

\section{Transcriptome analysis of HSV1-infected HaCaT cells}

RNA sequencing was performed in collaboration with BGI Europe Genome Center (Copenhagen, Denmark) following the standard operational procedure as described before(14). Briefly, the quality of total RNA was checked using the Agilent 2100 bioanalyzer. To construct the sequencing library for MGIseq-2000, approximately $1 \mu \mathrm{g}$ of polyA enriched RNA was used for library construction using the MGIEasy RNA Directional Library Prep Kit (MGI Tech). Next, paired-end sequencing with 100 cycles was performed using the MGISEQ-2000 sequencing instrument, according to the manufacturer's instructions. We generated an average of 63 million raw reads for each sample. The clean RNA reads were first aligned to the hg19 UCSC RefSeq (RNA sequences, GRCh37) using bowtie2 at first. To map the transcripts from the viruses, the unmapped reads were then aligned to the coding sequence of the human herpesvirus 1 (KOS strain). The expression of human genes and virus genes were performed by transforming mapped transcript reads to TPM using RSEM(15). The normalized expression were estimated and normalized by DEseq2. Differentially expressed genes were defined as genes with fold change over two-folds and adjusted P-value less than 0.001 using DESeq2.

Data availability.

RNA sequencing files generated for analysis of $\mathrm{HaCaT}$ cells infected with HSV in the presence or absence of 4-OI have been deposited to the data depository database (CNGBdb, https://db.cngb.org) with the following accession number : CNP0001039. 


\section{Ethics}

The project was approved by Institutional review boards at Aarhus University Hospital, by the Danish National Committee in Health Research Ethics (1-10-72-80-20) and the Danish Data protection Agency in accordance with the ethical standards of the Helsinki Declaration. Written informed consent was obtained from all study participants.

Statistical analysis.

Values were expressed as the means \pm SEM. Graphs and statistics were computed using Graph Pad Prism 7. An unpaired, two-tailed Student's $t$-test was used to determine significance of the difference between the control and each experimental condition. $P$ values of less than 0.05 were considered statistically significant, ***, $p<0.001 ; * \star, p<0.01$, and *, $p<0.05$.

\section{Methods References}

1. S. Matsuyama et al., Enhanced isolation of SARS-CoV-2 by TMPRSS2-expressing cells. Proc Natl Acad Sci U S A 117, 7001-7003 (2020).

2. D. Olagnier et al., Nrf2 negatively regulates STING indicating a link between antiviral sensing and metabolic reprogramming. Nature communications 9, 3506 (2018).

3. M. Hoffmann et al., SARS-CoV-2 Cell Entry Depends on ACE2 and TMPRSS2 and Is Blocked by a Clinically Proven Protease Inhibitor. Cell 181, 271-280 e278 (2020).

4. L. S. Reinert et al., TLR3 deficiency renders astrocytes permissive to herpes simplex virus infection and facilitates establishment of CNS infection in mice. J Clin Invest 122, 1368-1376 (2012).

5. C. L. Donald et al., Full Genome Sequence and sfRNA Interferon Antagonist Activity of Zika Virus from Recife, Brazil. PLoS Negl Trop Dis 10, e0005048 (2016).

6. L. Hilton et al., The NPro product of bovine viral diarrhea virus inhibits DNA binding by interferon regulatory factor 3 and targets it for proteasomal degradation. J Virol 80, 11723-11732 (2006).

7. H. D. Hoang et al., Induction of an Alternative mRNA 5' Leader Enhances Translation of the Ciliopathy Gene Inpp5e and Resistance to Oncolytic Virus Infection. Cell reports 29, 4010-4023 e4015 (2019).

8. K. L. Jonsson et al., IFI16 is required for DNA sensing in human macrophages by promoting production and function of cGAMP. Nature communications 8, 14391 (2017).

9. C. Chiang et al., Sequence-Specific Modifications Enhance the Broad-Spectrum Antiviral Response Activated by RIG-I Agonists. J. Virol. 89, 8011-8025 (2015).

10. M. I. Love, W. Huber, S. Anders, Moderated estimation of fold change and dispersion for RNA-seq data with DESeq2. Genome Bio/ 15, 550 (2014).

11. E. Y. Chen et al., Enrichr: interactive and collaborative HTML5 gene list enrichment analysis tool. BMC Bioinformatics 14, 128 (2013). 
12. D. Szklarczyk et al., STRING v11: protein-protein association networks with increased coverage, supporting functional discovery in genome-wide experimental datasets. Nucleic Acids Res 47, D607D613 (2019).

13. P. Shannon et al., Cytoscape: a software environment for integrated models of biomolecular interaction networks. Genome Res. 13, 2498-2504 (2003).

14. S. A. Jeon et al., Comparison of the MGISEQ-2000 and Illumina HiSeq 4000 sequencing platforms for RNA sequencing. Genomics Inform 17, e32 (2019).

15. B. Li, C. N. Dewey, RSEM: accurate transcript quantification from RNA-Seq data with or without a reference genome. BMC Bioinformatics 12, 323 (2011).

\section{Figures}
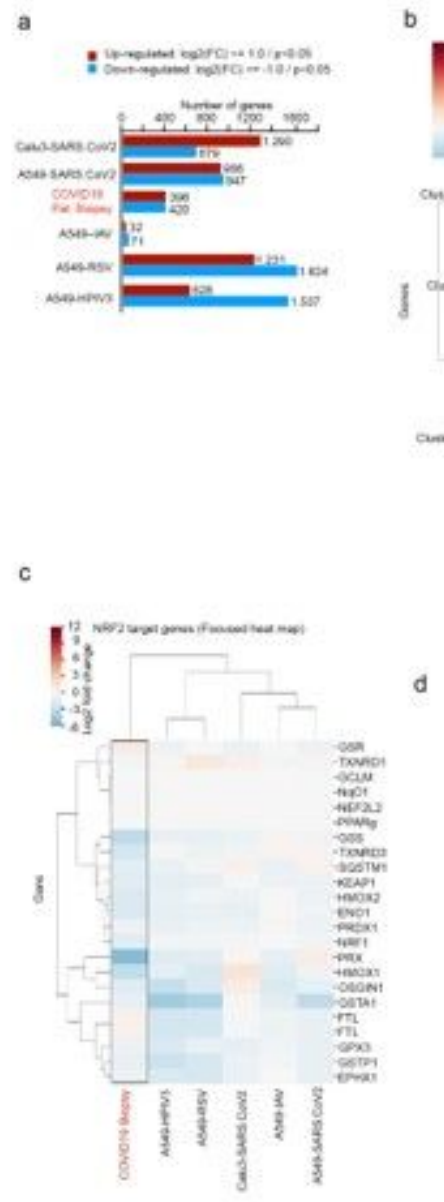

d
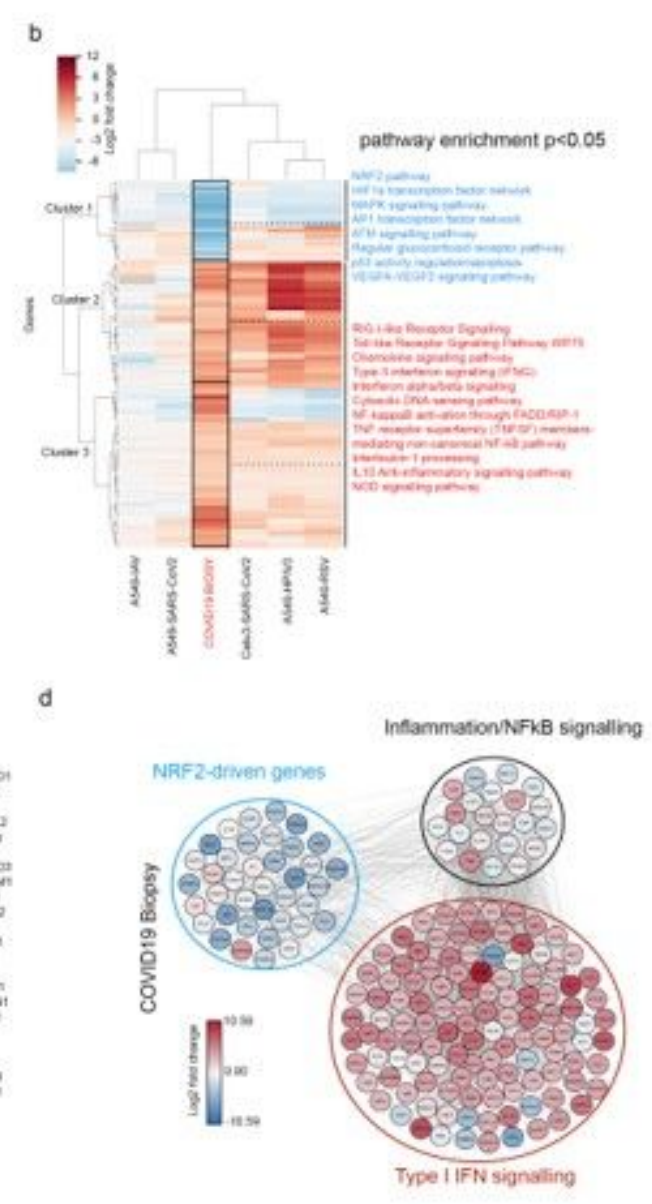

Figure 1 
Expression of NRF2-driven genes is suppressed in COVID-19 patient biopsies (a) Bar-chart of the number of transcripts that show differential expression (up and down). Genes with $p<0.05$ and Log2 fold change in COVID-19 patient lung biopsies were normalized against healthy lung biopsies, and in cell lines Calu3, NHBE and A549 infected with either SARS-CoV2, Influenza A virus (IAV), Respiratory Syncytial virus (RSV), or human parainfluenza virus type 3 (HPIV3) were normalized against mock treated cells. (b) Heat map of the subset of genes significantly differentially expressed in COVID-19 biopsies and simultaneously differentially expressed in at least 3 of the other conditions tested. The genes in each cluster was used for pathway enrichment analysis. Genes in cluster 1 are dominantly down regulated in COVID-19 biopsies. The genes in cluster 2 and 3 are up-regulated in biopsies and in most other test-samples. (c) heat map of NRF2 driven genes deferentially expressed in COVID-19 and in other conditions. (d) Cloud analysis of NRF2-driven differentially expressed genes. Subsets annotated as inflammation/NFロB signaling and Type I IFN signaling exhibit different expression patterns. The experiment is a re-analysis of data from BlancoMelo et al., https://doi.org/10.1101/2020.03.24.004655.

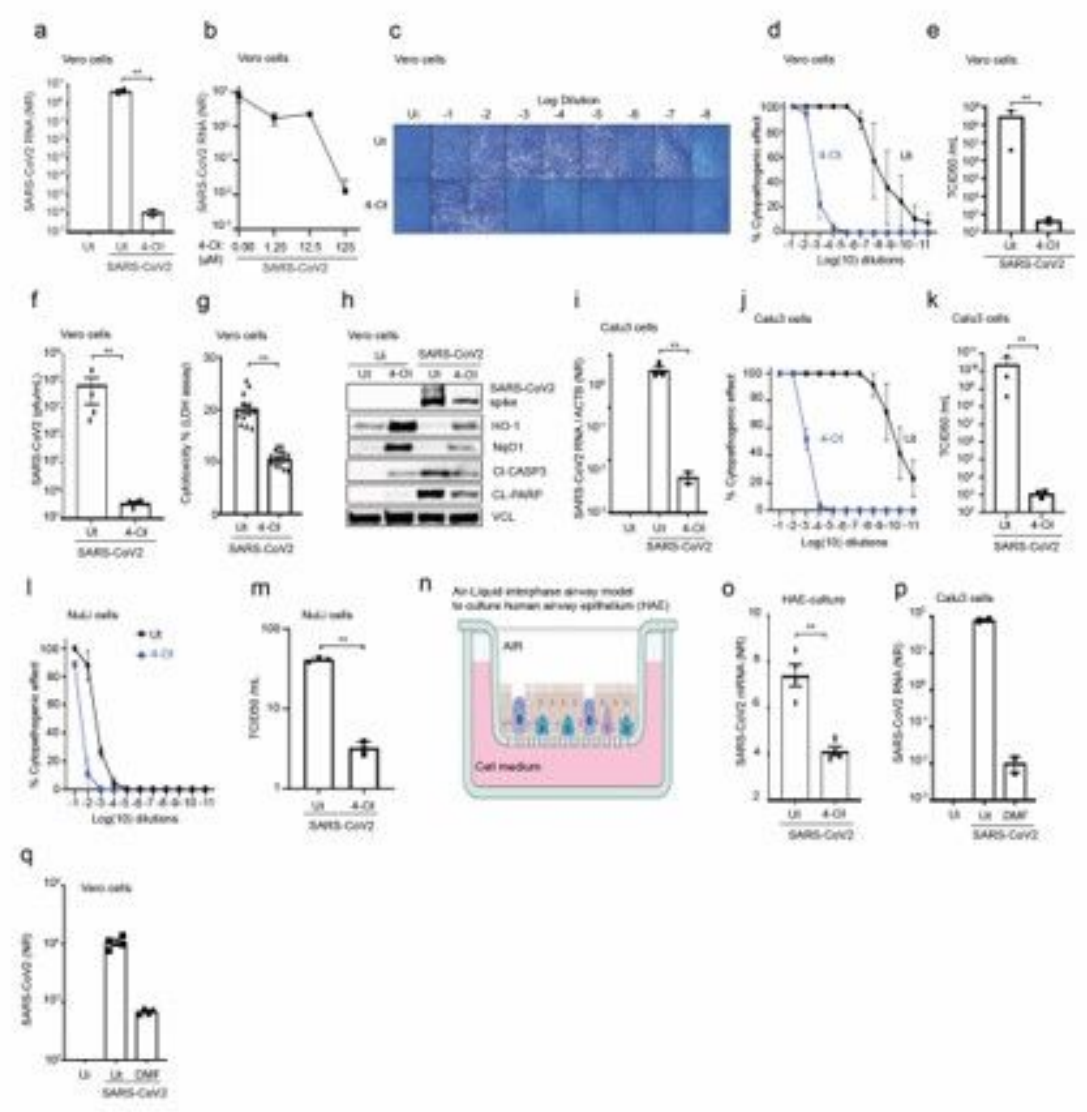

\section{Figure 2}

4-Octyl-itaconate (4-OI) and dimethyl fumarate (DMF) inhibit SARS-CoV2 replication (a-e) Vero E6 expressing human TMPRSS2 cells were treated with 4-OI (125ロM) or lower concentrations were indicated for $48 \mathrm{~h}$ and subsequently infected with SARS-CoV-2 at a $\mathrm{MOI}$ of 0.1 or 0.5 for $48 \mathrm{~h}$, respectively. Infection and replication were assessed by qPCR and TCID50 or plaque assay, respectively. Graphs in $(a+b+e)$ 
display means and s.e.m. of one experiment performed in duplicates. (f) Supernatants from (e) were also assessed for viral titers by semi-solid agarose plaque assay. From (a-f) A two-tailed Student's t-test was used to determine statistical significance where ${ }^{*} \mathrm{p}<0.01$. (g) Vero E6 expressing human TMPRSS2 cells were treated with $4-\mathrm{Ol}(125 \square \mathrm{M})$ for $48 \mathrm{~h}$ and subsequently infected with SARS-CoV-2 at a MOI of $0.148 \mathrm{~h}$. Cell death was evaluated using an LDH release assay. Data are the means and s.e.m. of one experiment performed in sextuplicate where ${ }^{* *} \mathrm{p}<0.01$ has been determined by a two-tailed Student's t-test. (h) Whole cell extracts from (g) were immunoblotted for various Nrf2 and death associated markers (i-k) Calu3 cells were treated with 4-OI (100ロM) for 48h and subsequently infected with SARS-CoV-2 (MOI 0.5) for 48h. Viral RNA content and progeny virus were assessed by GPCR and TCID50, respectively. Data are the means and s.e.m. of one experiment performed in triplicate where ${ }^{* *} p<0.01$ has been determined by a two-tailed Student's t-test. (I-m) NuLi cells were treated with 4-OI (100ロM) for 48h and subsequently infected with SARS-CoV-2 ( $\mathrm{MOI} 0.5$ ) for $48 \mathrm{~h}$. Viral titers were determined by TCID50. Data are the means and s.e.m. of one experiment performed in duplicate where ${ }^{*} \mathrm{p}<0.01$ has been determined by a two-tailed Student's t-test. (n) schematic of an HAE culture. (o) HAE cultures $(n=4)$ were treated with 4-OI $(125 \square M)$ overnight and were subsequently infected with SARS-CoV-2 (MOI 0.1). Viral genome content was assessed by qPCR (mean +/- s.e.m. from 4 independent primary HAE cultures). (p-q) Calu-3 cells and Vero E6 expressing hTMPRSS2 cells were treated with dimethyl fumarate (DMF) (200 $\mathrm{MM})$ for $48 \mathrm{~h}$ and subsequently infected with SARS-CoV-2 at a MOI of 0.5 and 0.1 , respectively. Data are the means and s.e.m. of one experiment performed in duplicate where ${ }^{* *} p<0.01$ has been determined by a two-tailed Student's t-test.

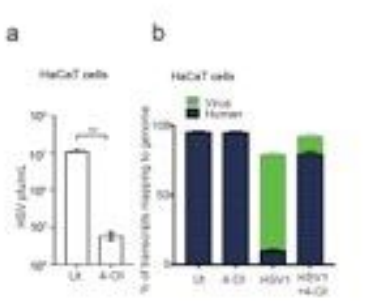

f

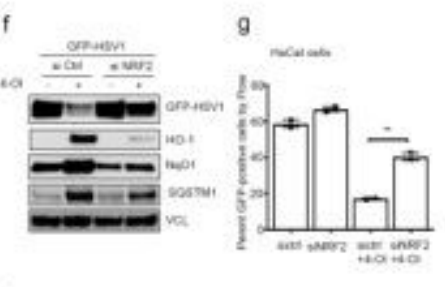

k

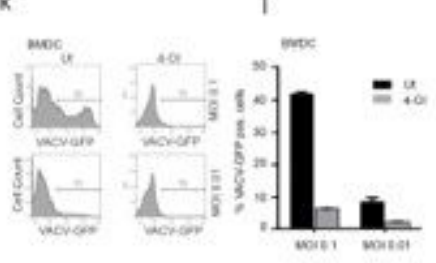

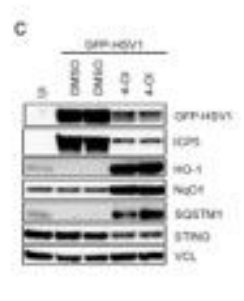
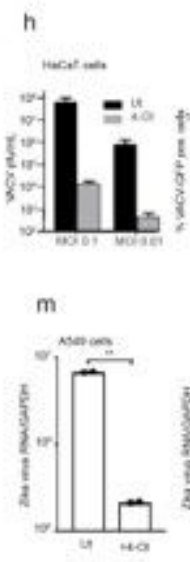
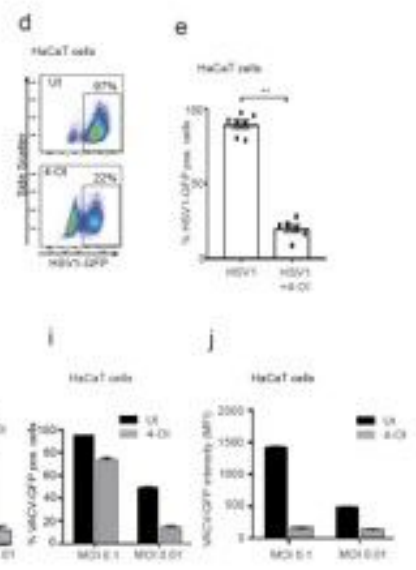

n

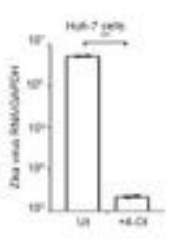

Figure 3 
4-OI broadly inhibits other pathogenic viruses including HSV, VACV, and Zika Virus (a) HaCaT cells treated with 4-OI (125ロM) for $48 \mathrm{~h}$ and infected with HSV1-GFP (MOI 0.01). Viral titers were determined by plaque assay. Data are representative of one experiment performed in duplicates where ${ }^{* *} p<0.01$ has been determined by a two-tailed Student's t-test. (b) RNA analyzed using RNA-sequencing ( $n=3)$. (c-e) HaCaT cells treated with 4-OI (125ロM) and infected with HSV1-GFP (MOI 0.01). Lysates were analyzed by immunoblotting with Vinculin (VCL) as loading control and by flow cytometry $(n=7$ from three independent experiments, mean +/- s.e.m.). (f-g) HaCaT cells were lipofected with siRNA for $72 \mathrm{~h}$, subsequently challenged with 4-OI (125ロM) before HSV1-GFP infection (MOI 0.01) for 24h. Infectivity and silencing efficiency was determined by immunoblotting (f) and flow-cytometry (g). (h-l) HaCaT cells (h-j) and BMDCs $(\mathrm{k}-\mathrm{l})$ were treated with $4-\mathrm{OI}(125 \square \mathrm{M})$ for $48 \mathrm{~h}$ and infected with VACV for $24 \mathrm{~h}$. Viral titers and infectivity were determined by plaque assay (h) and flow cytometry (i-l). (m-n) A549 and Huh-7 cells were pre-treated with 4-OI for 48h (150!M) and infected with Zika virus (ZIKV) (MOI 0.1) for 4 days. Viral genome was determined by qPCR. Data are representative on one experiment performed in duplicates where means and s.e.m are displayed and ${ }^{* *} \mathrm{p}<0.01$ has been determined by a two-tailed Student's t-test.
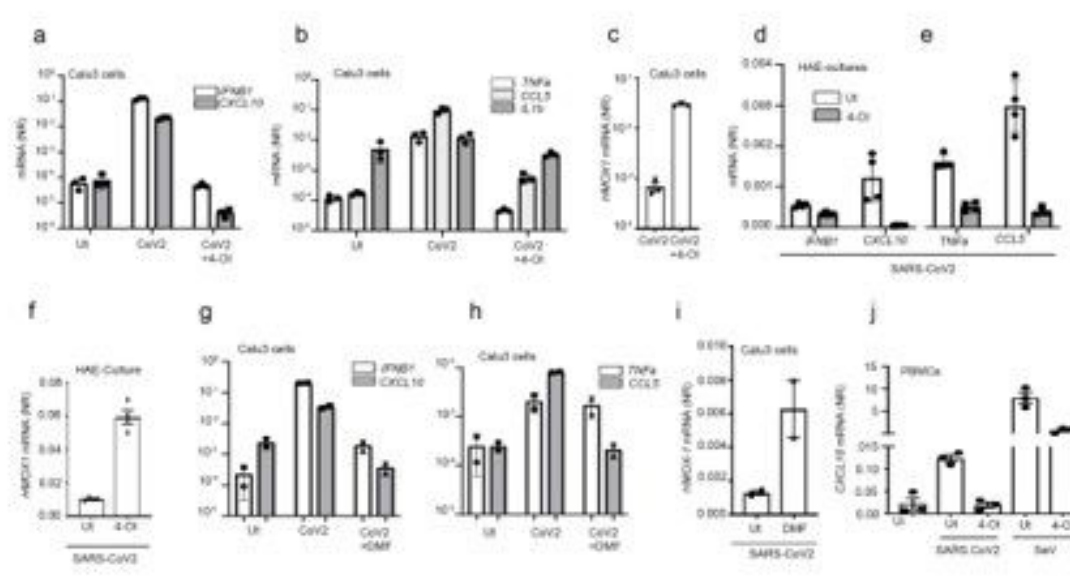

h
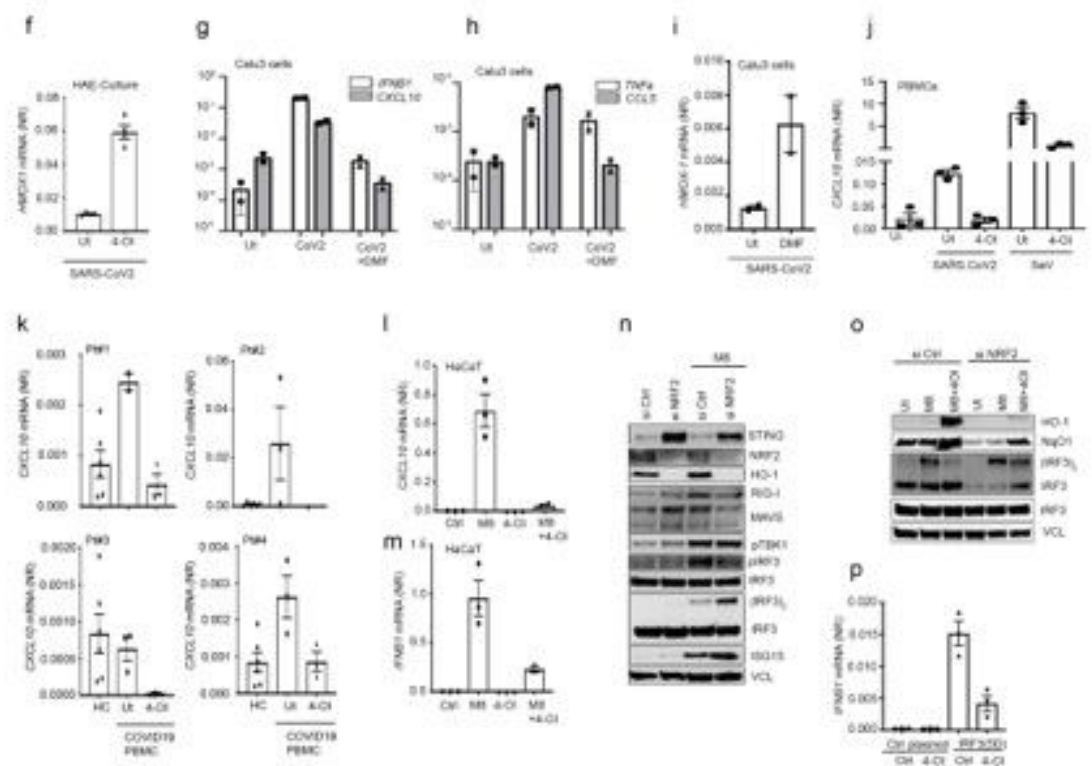

$\circ$
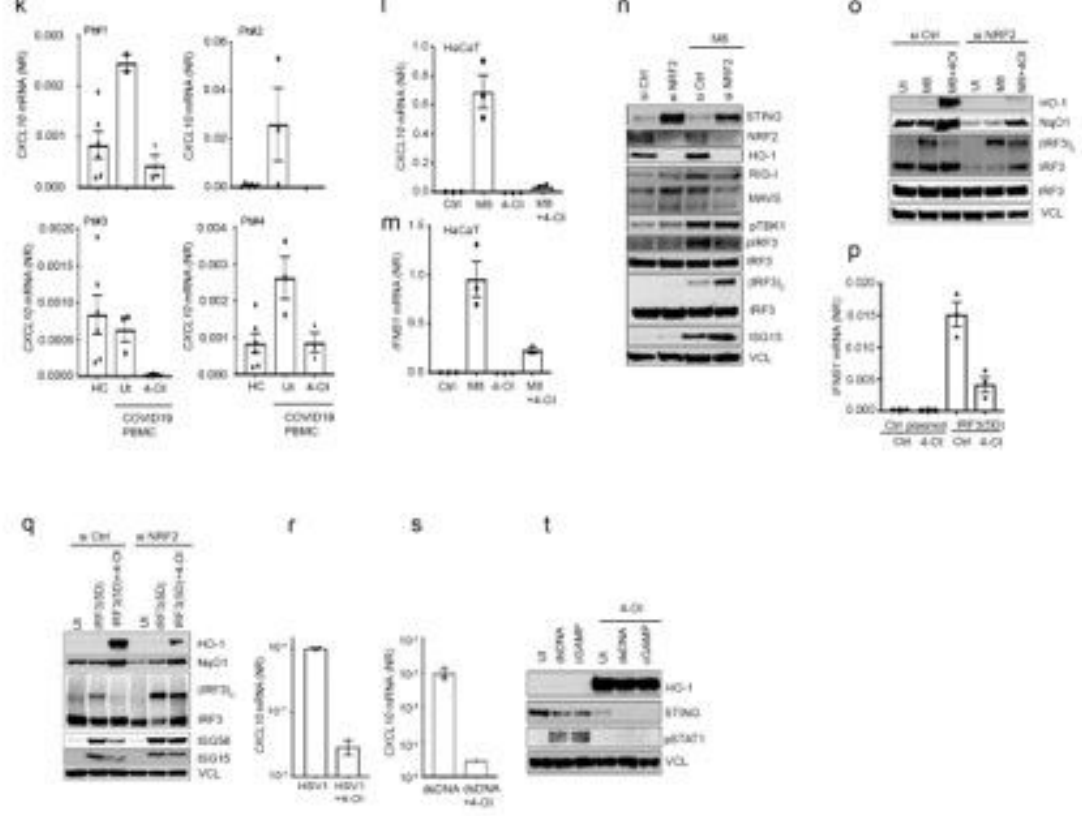


\section{Figure 4}

4-OI and DMF limit SARS-CoV2 and HSV induced inflammatory responses. (a-c) Calu-3 treated with 4-OI $(125 \mu \mathrm{M})$ for $48 \mathrm{~h}$ before infection with SARS-CoV2 (MOI 0.5) for 48 hours. RNA was extracted for analysis by qPCR. (d-f) HAE cultures $(n=4)$ were treated overnight with $4-0$ l at $125 \mu \mathrm{M}$ before SARS-CoV2 infection (MOI 0.1) for 24 and analysis by qPCR. (g-i) Calu-3 treated with DMF $(200 \mu \mathrm{M})$ for $48 \mathrm{~h}$ before infection with SARS-CoV2 (MOI 0.5) for 48 hours. RNA was extracted for analysis by qPCR. In $(a+b+c+g+h+i)$ data are representative of one experiment performed in duplicates where means and s.e.m. are displayed. In (d-f) data are representative of four independent primary HAE cultures where means and s.e.m. are displayed. (j) Healthy PBMCs were pre-treated overnight with 4-OI $(100 \mu \mathrm{M})$ before a challenge with SARSCoV-2 (MOI 10) or sendai virus (SeV) (50 HAU) for an additional 24h. CXCL10 mRNA levels were determined by qPCR. Data are representative of one healthy donor in triplicate. Experiment has been performed on two independent healthy donors. (k) PBMCs from four COVID-19 patients ( $k$ ) and 2 healthy controls $(\mathrm{HC})$ treated with $4-\mathrm{Ol}$ at $100 \mu \mathrm{M}$ overnight before analysis by qPCR. (I-m) HaCaT cells were treated with 4-OI $(125 \mu \mathrm{M})$ before stimulation with the sequence optimized RIG-I agonist M8 $(10 \mathrm{ng} / \mathrm{mL})$ for 6 hours followed by qPCR gene expression analysis. Data represent the means and s.e.m. of one experiment performed in triplicate. ( $\mathrm{n}$ ) HaCaT cells were lipofected with indicated siRNAs for $72 \mathrm{~h}$ before treatment with M8 $(10 \mathrm{ng} / \mathrm{mL})$ for 3 hours followed by analysis by immunoblotting. (o) HaCaT cells were lipofected with indicated siRNAs for $72 \mathrm{~h}$ before treatment with $4-\mathrm{Ol}(125 \square \mathrm{M})$ for $48 \mathrm{~h}$ and stimulation with M8 $(10 \mathrm{ng} / \mathrm{mL})$ for 3 hours followed by analysis by immunoblotting. (p-q) HEK293(p) and HaCaT(q) cells were transfected with indicated plasmids before treatment with $4-\mathrm{Ol}$ at $125 \mu \mathrm{M}$. In (q), HaCaT cells were lipofected with siRNAs for $72 \mathrm{~h}$ before plasmid transfection. Cells were then collected for analysis by

qPCR (p) and immunoblotting (q). (r-t) HaCaT cells were treated with 4-Ol at $125 \mu \mathrm{M}$ before infection with HSV1 at MOI 0.01 or transfection with dsDNA (4 घg.mL-1). Cell pellets were collected for qPCR and immunoblotting at 6 and 3 hours respectively.

\section{Supplementary Files}

This is a list of supplementary files associated with this preprint. Click to download.

- Supplementalfigures.pdf 